Cells agglutinated by the antibody form characteristic large rather loose masses. Lewis-positive cells which have been treated with a barely agglutinating dose of antibody and washed are not agglutinated by anti-human-globulin serum ${ }^{2}$. The antibody thus differs from the $R h$ antibodies and apparently resembles in this respect the isoagglutinins $a$ and $\beta^{2}$.

I should like to acknowledge the assistance which $I$ have received from Mr. R. Hudson of the N.E. London Blood Supply Depot, Luton who flrst found and directed my attention to these antibodies, and
from Dr. R. R. Race. A full account of the investigation will be published elsewhere.

Blood Group Reference Laboratory,

A. E. MoUrani

c/o Lister Institute of Preventive Medicine,

Chelsea Bridge Road,

London, S.W.1.

July 5.

1 Callendar, Race and Paykoc, Brit. Med. J., ii, 83 (1945).

: Coombs, Mourant and Race, Lancet, i, 264 (1946).

Combined Action upon Muscle of Adenosine Triphosphate, Acetylcholine and Potassium, Calcium and Magnesium lons

IN a note published in Nature in 1944, Buchtal and Kahlson' have pointed out that the close intra-arterial injection of $5 \mu \mathrm{gm}$. acetylcholine after introduction of adenosine triphosphate increases the intensity and duration of the mechanical response of muscle. The combined action of these substances is of considerable interest, in view of the important part taken by adenine triphosphate in muscular contraction and of the participation of acet

of impulses at the myoneural junction. the experiments have been carried ont with the dorsal muscles were placed in Ringer solution of the following composition: sodium chloride, $6.0 \mathrm{gm}$. ; potassium chloride. $0.14 \mathrm{gm}$. ; calcium chloride, 0.06-0.1 gm. ; sodium bicarbonate, $0.1 \mathrm{gm}$.; and water, 1 itre. The sodium salt of adenosine triphosphate was prepared immediately before the experiment and used in concentrations of $1 \times 10^{-3}-$ $1 \times 10^{-4}$ with frog muscles and in concentrations of $2 \times 10^{-4}-$ $1 \times 10^{-6}$ with dorsal leech muscles.

It was found that these concentrations of adenosine triphosphate did not by themselves produce any contraction of the $\mathrm{m}$. rectus abdominis of the frog, and they led to small contractile response of the leech muscle. After one application of the adenosine triphosphate solution the frog and leech muscles react to solutions of acetylcholine with contractions of increased intensity. The contractions remain increased when the solution of acetylcholine is applied several times. It follows that adenosine triphosphate sensitizes the muscle to acetylIt follows that adenosine triphosphate sensitizes the muscle to acetyl-
choline. The initial smaller contractile effect of acetylcholine is restored only after repeated washing of the muscle with Ringer solution.

In another series of experiments, we investigated the contractile response of muscle to the action of adenosine triphosphate and acetylresponse of muscle to the action

These experiments were undertaken in view of the fact that potassium, calcium and magnesium ions influence the activity of adenosinetriphosphatase $\mathrm{e}^{2-5}$ and the contraction of myosin threads 8.7 . According to these data, calcium ions activate adenosine triphosphatase, thereby promoting its breakdown, and potassium and magnesium ions inhibit promoting its breakdown, and potassium and magnesium ions inhibit adenosine triphosphate. On the other hand, it has been shown that the adenosine triphosphate. On the other hand, it has been shown that the contraction of myosin threads produced by adenosine triphosphate in the presence of potassium chloride is inhibited

To determine the effect of changed ionic environment we have used Ringer solution with increased contents of calcium chloride (up to $0.3 \mathrm{gm}$. in $1 \mathrm{l}$.) or of potassium chloride (up to $0.28 \mathrm{gm}$. in 11 .) ; in some experiments magnesium chloride was added to the Ringer solution $(0.1 \mathrm{gm}$. to 11 .). The increased concentration of potassium ions leads to a markedly increased reaction to acetylcholine of muscles subjected to the action of adenosine triphosphate. This increased
sensitivity to acetylcholine persists longer than in normal Ringer solution. The same effect is obtained in the presence of magnesium

The opposite effect was observed under the influence of calcium ons. When their concentration in the solution is increased, adenosine triphosphate does not cause contraction of the dorsal muscle of the leech; sensibilization to acetylcholine was also absent, and the muscle was less responsive to it. Both in the frog muscle and in that of the leech adenosine triphosphate produces no contraction in Ringer solution with increased content of calcium, and eads to a persistent decrease of the contractile response to acetylcholine. Our data on the action of potassium, magnesium and calcium ions upon the contractile response of muscle produced by adenosine triphosphate coincide with the results obtained by Szent-Györgyi and Erdös with myosin threads. They show that the action of ions upon adenosine triphosphatase and their action upon contractility of muscle are two separate and not directly parallel processes.

Physiological Iaboratory,

EUG. B. BABSK

Institute of Biological Chemistry,

Academy of Medicine, Moscow.

1 Buchtal, F., and Kahlson, G., Nature, 154, 178 (1944).

Ljubimova, N., and Pevzner, D., Biochim., 6, 178 (1941).

- Bailey, K., Biochem. J., 36, 121 (1942).

Mehl and Sexton, Proc. Soc. Exp. Biol. and Med., 52, 38 (1943)

Banga, J., and Szent-Györgyi, A., Stud. Inst. Med. Chem. Univ. Szeged, 3, 72 (1943)

Szent-Györgyi, A., Acta Physiol. Scand., 9, suppl. 25 (1945).

- Erdös, T., Stud. Inst. Med. Chem. Univ. Szeged, 1 (1942).

\section{Role of Thiamin and Riboflavin in the Biosynthesis of Vitamin C}

THE rat is known to synthesize vitamin $\mathrm{C}$ in the course of its normal metabolism. It has been observed by Longenecker and associates that this synthesis can be greatly stimulated, resulting in the high urinary excretion of the vitamin, when the rats are given daily a
variety of compounds, particularly narcotics. In view of the great variety of compounds, particularly narcotics. In view of the great variation in the nature and constitution of the effective compounds, these authors are inclined to the view that these substances act as promoters rather than precursors for the synthesis of the vitamin.
They were not able to suggest, however, any probable mechanism for this biosynthesis of vitamin $\mathrm{C}$ by narcotized rats.

It has been observed by us that narcotics such as chloretone stimulate the synthesis of vitamin $\mathrm{C}$ in rats without signiflcantly affecting urinary sugar, nitrogen and phosphorus, blood sugar and phosphoru and liver glycogen. Injection of intermediary metabolites such as pyruvate, lactate, etc., into rats has no effect on the synthesis. The process has also been found to be independent of certain endocrine factors, as the excretion under the narcotic remains unaffected by adrenalectomy or pancreatectomy.

Different dehydrogenase systems such as citric, glutamic, glycerophosphoric, succinic, glucose, lactic and pyruvic, present in rat tissues, have been studied by the methylene blue technique. It has been observed that pyruvic and lactic dehydrogenases are affected in the chloretonized condition of the animals. The effect of chloretone on the aerobic and anaerobic oxidation of pyruvate by brain tissue of the rat under normal and narcotized conditions of the animals has been studied by the Warburg manometer and by the ferricyanide technique of Quastel et al. ${ }^{2}$ respectively. A deflnite inhibition of pyruvic acid oxidation has been observed under aerobic condition, the effect being far less pronounced in the anaerobic state. This seems to indicate, as also suggested by Michaelis et al. ${ }^{3}$, that chloretone exerts its main effect on that part of the system which is responsible for the oxidation of pyruvic acid under aerobic condition, and not on the components capable of oxidizing it anaerobically.

Following up this study further, it has been observed that the stimulating effect of chloretone on the biosynthesis of vitamin $\mathrm{C}$ by the rat and the consequent urinary excretion of the vitamin can be almost completely suppressed, if the animals are made thiaminalmost completely suppressed, if the animals are made thiamin-
deflcient. This stimulating effect can be restored to the deficient deficient. This stimulating effect can be restored to the deficient week, the sparing action of fat on thiamin and the possibility of vitamin week, the sparing action of fat on thiamin and the possibility of vitamin $\mathrm{C}$ being destroyed during the abnormal state of vitamin $\mathbf{B}_{1}$-deflciency
of the rats have also been considered. It has been observed that the of the rats have also been considered. It has been observed that the
stimulating influence of chloretone on the synthesis of vitamin C stimulating influence of chloretone on the synthesis of vitamin c by the rat is not affected by these conditions of the animals. It appears therefore that thiamin has some speciflc role in the synthesis pyruvic acid oxidation by the narcotic is very probably also a factor prolved in the process.

It has further been found that the stimulating effect of chloretone on the synthesis of vitamin $C$ and its urinary excretion by the rats can also be greatly suppressed if the animals are made riboflavin-deficient. also be greatly suppressed if the animals are made riboflavin-deficient. administering riboflavin to the deflcient rats. It has also been shown by the paired feeding technique that inanition does not affect the role of riboflavin in this synthesis of ascorbic acid by chloretonized rats.

Barron et al. ${ }^{4}$ have shown that thiamin or its phosphorylated form diphosphothiamin is closely associated with pyruvic acid metabolism
in the living organism. Ball pyruvic acid metabolism in which both thiamin pyrophosphate and lavoprotein are involved. From the present work it would appear that if pyruvic acid is transformed into ascorbic acid in the narcotized rat, this

Our thanks are due to Dr. S. Banerjee for the surgical operation of some animals in connexion with the investigation. We are also in debted to the Indian Research Fund Association for a grant. Gratefu acknowledgment is also made to Messrs. Hoffmann-La Roche, New Jersey, for the gift of the vitamins used in this work.

S: C. ROY

B. C. GUHA

Department of Applied Chemistry

University College of Science and Technology, July 11 .

${ }^{1}$ Longenecker, H. E., Fricke, H. H., and King, C. G., J. Biol. Chem., $135,497(1940)$

2 Quastel, J. H., and Wheatley, A. H. M., Biochem. J., 32, 1936 (1938). - Michaelis, M., and Quastel, J. H., Biochem. J., 85, 518 (1941). J. Biol. Chem., 141, 957 (1941).

$\checkmark$ Ball, E. G., Cold Spring Harbor Symposia Quant. Biol., 7, 100 (1939).

\section{Production of Potent Botulinum Toxins and Formol-Toxoids}

THE preparation of suitable botulinus vaccines requires the production of toxin of great potency and high antitoxin combining power. The process of detoxication with formalin is facilitated if the medium is low in inactive proteinaceous substances. One of us (A. P.) suggested growing botulinus in 'Cellophane' bags flled with saline and immersed in meat broth (broth with meat particles). The bacilli grew readily in the bags and gave a very high yield of toxin. 'Cellophane' bag that contained nutrient broth instead of saline, gave little toxin. A 'D' type strain grown in saline in 'Cellophane' bags gave a culture
of which $10^{-8}$ c.c. killed a mouse. The $L f$ and $L+$ were about eighty of which $10^{-8}$ c.c. killed a mouse. The Lf and Lt were about eighty
times those found with cultures grown in the ordinary way. The times those found with cultures grown in the ordinary way. The fltrate was readily detoxicated in $7-10$ days with 0.5 per cent formalin, 